\title{
Tuberculous Hypertrophic Pachymeningitis in A 10 Years Old Girl: Report and A Review of the Literature
}

Zouita B*, Bouyaali C, El badri Y, El Hadri K, Basraoui D, Jallal H

The Mother-Child Hospital, University Hospital Mohammed VI Marrakech

DOI: $10.36347 /$ sjmcr.2020.v08i04.010

| Received: 31.03.2020 | Accepted: 08.04.2020 | Published: 11.04.2020

*Corresponding author: Zouita B

Abstract

Case Report

Pachymeningitis is a rare disease of diverse etiology mainly affecting the adult population. And very rare pediatric cases have been reported till now. We report the young child with pachymeningitis. Our case responded very well to antitubercular therapy with near complete recovery. Antitubercular therapy can be considered in children from endemic countries with hypertrophic pachymeningitis before labeling their condition as idiopathic hypertrophic pachymeningitis.

Keywords: Tuberculous Pachymeningitis Antitubercular.

Copyright @ 2020: This is an open-access article distributed under the terms of the Creative Commons Attribution license which permits unrestricted use, distribution, and reproduction in any medium for non-commercial use (NonCommercial, or CC-BY-NC) provided the original author and source are credited.

\section{INTRODUCTION}

Even in tuberculosis prevalent countries, the diagnosis of tuberculosis meningitis is difficult at times and requires a high degree of clinical suspicion. Direct cerebrospinal fluid examination and polymerase chain reaction of CSF (TB-PCR) are specific diagnostic tools but have varied sensitivities, and mycobacterial culture is time-consuming and can delay timely diagnosis [1].

Cranial pachymeningitis is a rare disorder in which there is diffuse or localized thickening of the cranial dura mater, with or without associated inflammation. Patients commonly present with manifestations of increased intracranial pressure, such as headache, or progressive neurologic deficits arising due to compression of neural structures by the thickened dura mater [2]. While a number of causes of dura mater thickening have been reported, including trauma, infection, autoimmune diseases, connective tissue diseases, sarcoidosis, and malignancy, many cases of cranial pachymeningitis are thought to be idiopathic [1]. The finding that some idiopathic cases show a beneficial response to antituberculous therapy suggests, however, that a proportion of them may be caused by tuberculosis [3, 4].

\section{CASE}

A 7 year old female child presented one year ago her hospitalization a headache, and behavioral disorder with vomiting induced by insertion of the finger into the mouth, causing undernutrition with cachexia, at june 2019 Initial CT brain scan suspected a left frontal hematoma probably depends on a Sylvien artery, completed with the first brain MRI at the same month suspected a arteriovenous malformation, completed with brain angiography wish is normal. She was hospitalized in January 2020 at the university hospital at Marrakech for etiological assessement.

At admission, he presented a several hypotrophy (Gomez score at $52 \%$, WATERLOW 98 $\%)$,

Anemia with hemoglobin at $10,8 \mathrm{~g} / \mathrm{dL}$, leukocytes at 9650 elements/uL. Liver function, renal function, ionogram, and chest radiograph were normal. Quantiferon was negative, and Cerebrospinal fluid analysis revealed sterile, number of cell in it is low than3 elements, Glucose level was $0,22 \mathrm{~g} / \mathrm{L}$, and protein was $8,74 \mathrm{~g} / \mathrm{L}$. Cerebrospinal fluid for bacterial antigens and tuberculosis, was negative. Blood and cerebrospinal fluid cultures were sterile.

The second Magnetic resonance imaging (MRI) of brain realized on 24 February 2020 revealed multifocal nodular abnormalities of white matter under bilateral matter frontal and parietal cortex, and periventriculaire in $\mathrm{T} 2$ and FLAIR hyper signal, meningeal thikening nodular and diffuse linear contrast and enhancement with more pronounced in the left basi frontal, temporal, perimedullary and sheath of the V, II nerves and optic chiasma, associated with compression 
of left lateral sinus, proposing tuberculous hyperthrophic pachymeningitis.

The diagnosis of meningeal tuberculosis was retained, with introduction of anti-bacillary treatment
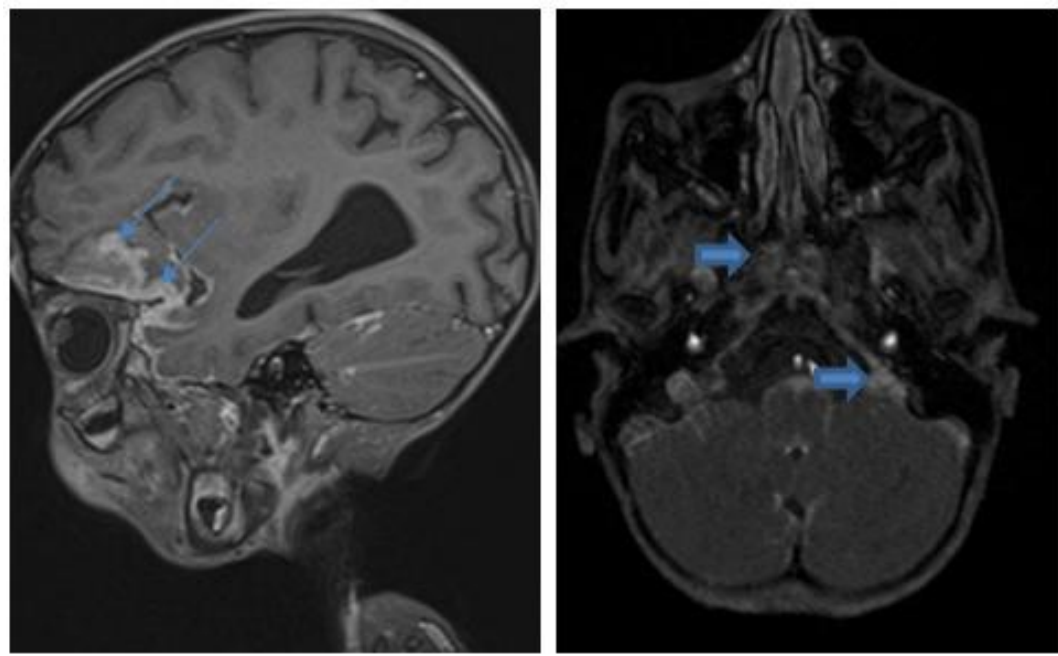

Fig-1 :3D T1-weighted postcontrast magnetic resonance imaging meningeal thickening, nodular and diffuse linear contrast and enhancement with more pronounced in the left basi frontal, perimedullary and sheath of the $\mathrm{V}$, II nerves and optic chiasma(arrows)
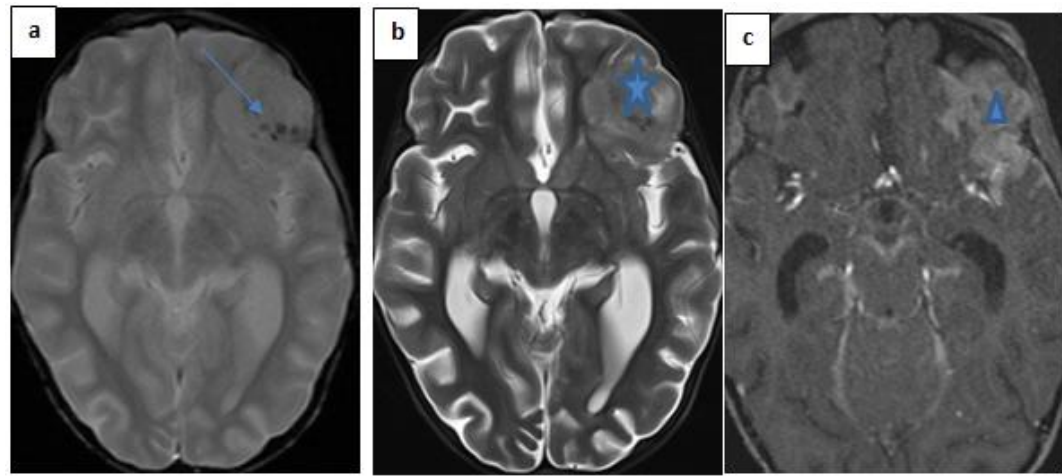

Fig-2: Axial T2*(a)axial T2 (b) and T1 postcontrast weighted magnetic resonance imaging reveals a left frontal lobe pseudo-mass (star)isointense relative to gray matter and contains scattered hypointense foci(arrow) with intense enhancement (triangle)
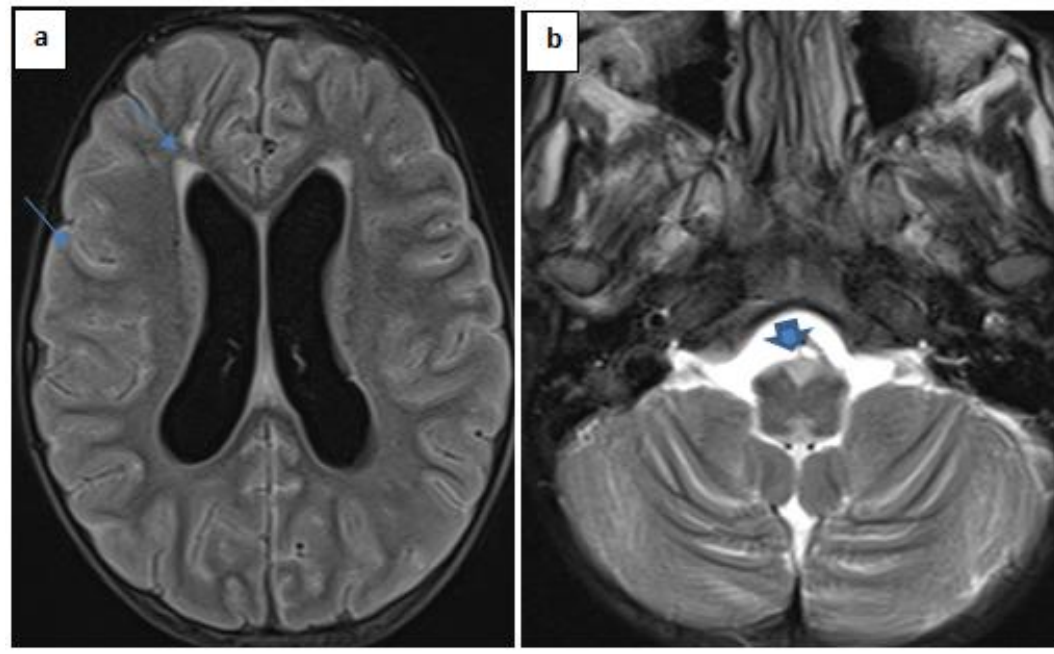

Fig-3 : Axial FLAIR(a) and axial T2(b) weighted MRI image : shows hyperintense multifocal nodular abnormalities of white matter under bilateral matter frontal and parietal cortex, and péri-ventriculaire hyper signal (arrows) associated with leptomeningeal thinking hyper intense on T2(arrow) 


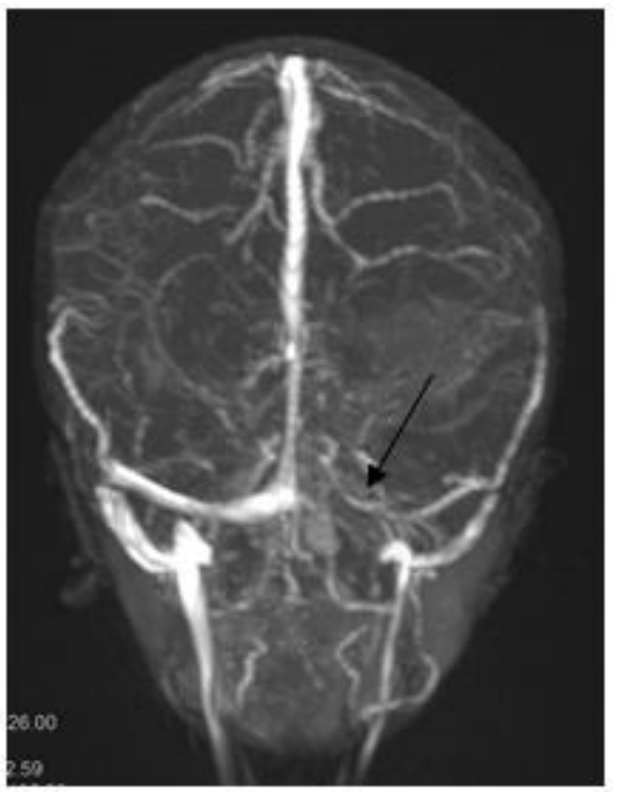

Fig-1: Angiomri Tof : compression of left lateral sinus (arrow)

\section{DISCUSSION}

Hypertrophic pachymeningitis was first described by Charcot and Joffroy in 1869. This rare disorder is characterized by inflammation and thickening of dura mater of diverse etiology. Infective cause includes tubercular, syphilis, fungal, cysticercosis, pseudomonas, Lyme disease, and human T-cell lymphotrophic virus infection [5]. It also has been reported with malignancy, after head trauma, intracranial hypotension, and autoimmune syndromes like rheumatoid arthritis, Wagner granulomatosis, jogren syndrome, sarcoidosis, Takayasu, and temporal arteritis [6, 7]. Although idiopathic variety is seen in most cases, efforts should be made to delineate the other treatable cause. Tuberculosis in particular in our context; Clinical features usually include headache, cranial nerve palsy, and ataxia. Other features include focal neurologic deficit, dural venous sinus occlusion, internal carotid artery occlusion, intracranial hemorrhage, and obstructive hydrocephalus. Mean age of presentation is 51 years, with a range from 3 to 78 years.

Review of case of hypertrophic pachymeningitis revealed that fever is usually not present, cerebrospinal fluid is normal in one-fourth of cases, two-thirds revealed high protein, and one-fourth have lymphocytic pleocytosis. In the absence of definitive evidence of tuberculosis, antitubercular therapy was started. In another pediatric case where no definitive diagnosis of tuberculosis could be established and empirical antitubercular therapy was started. This patient responded well.

Neuroimaging is essential in making the diagnosis. Contrast material-enhanced computed tomography (CT) reveals diffuse thickening and enhancement of the dura mater [8,9]. It is well known that gadolinium enhanced MR imaging is superior to contrast enhanced CT in the evaluation of leptomeningeal disease. Findings of infiltrating meningeal lesions can be subtle at unenhanced MR imaging, and lack of contrast enhanced imaging is the primary cause of delayed diagnosis [8]. MR imaging should include T1- weighted, T2-weighted, and gadolinium-enhanced T1-weighted sequences in multiple projections. Typically, hypethrophic meningitis demonstrates smooth or nodular dural thickening that is isointense or hypointense with both T1- and T2-weighted sequences. It also shows avid enhancement after intravenous administration of contrast material. These signal intensity characteristics are due to the fibrosis and necrosis of the dura mater [10-13]. Peripheral hyperintensity can be seen on T2weighted images and is thought to represent active inflammation or increased vascularity of the dura mater and underlying parenchyma. However, in the present case, MR imaging findings were somewhat atypical for IHP, with tumefactive enlargement of the dura mater mimicking a vascular lesion. However, even though IHP can manifest as a dural mass mimicking a meningioma [9, 14], there are few documented cases in the literature. Angiography typically reveals an avascular mass [14]

\section{REFERENCES}

1. Baker CA, Cartwright CP, Williams DN, Nelson SM, Peterson PK. Early detection of central nervous system tuberculosis with the Gen-Probe nucleic acid amplification assay: utility in an inner city hospital. Clinical infectious diseases. 2002 Aug 1;35(3):339-42.

2. Kupersmith MJ, Martin V, Heller G, Shah A, Mitnick HJ. Idiopathic hypertrophic pachymeningitis. Neurology. 2004;62:686-694.

3. Goyal M, Sharma A, Mishra NK, Gaikwad SB, Sharma MC. Imaging appearance of pachymeningeal tuberculosis. AJR Am J Roentgenol. 1997;169:1421-1424.

4. Parney IF, Johnson ES, Allen PB. "Idiopathic" cranial hypertrophic pachymeningitis responsive to antituberculous therapy: case report. Neurosurgery. 1997;41:965-971.

5. Bhatia R, Tripathi M, Srivastava A, Garg A, Singh MB, Nanda A, Padma MV, Prasad K. Idiopathic hypertrophic cranial pachymeningitis and dural sinus occlusion: two patients with long-term follow up. Journal of Clinical Neuroscience. 2009 Jul $1 ; 16(7): 937-42$

6. Van Toorn R, Esser M, Smit D, Andronikou S. Idiopathic hypertrophic cranial pachymeningitis causing progressive polyneuropathies in a child. Eur J Paediatr Neurol. 2008;12: 144-147.

7. Karimi A, Tabatabaei SR, Shiva F. Tuberculous pachymeningitis in a young child with spinal involvement: a case report. Arch Pediatr Infect Dis 2012;1:36-39. 
8. Rukhsana Tariq R, Rashid Ahmed R. Tuberculous hypertrophic pachymeningitis presenting as visual blurring and headaches. J Pak Med Assoc. 2012;62:966-968.

9. Wang YJ, Fuh JL, Lirng JF, Lu SR, Wang SJ. Headache profile in patients with hypertrophic cranial pachymeningitis. Headache. 2004; 44:916923.

10. Kioumehr F, Rooholamini SA, Yaghmai I, Verma R. Idiopathic hypertrophic cranial pachymeningitis: a case report. Neuroradiology. 1994; 36:292- 294.

11. Riku S, Kato S. Idiopathic hypertrophic pachymeningitis. Neuropathology. 2003; 23:335344
12. Nishioka H, Ito H, Haraoka J, Yamada Y, Nojima H. Idiopathic hypertrophic cranial pachymeningitis with accumulation of thallium-201 on singlephoton emission CT. AJNR Am J Neuroradiol. 1998; 19:450-453.

13. Bang OY, Kim DI, Yoon SR, Choi IS. Idiopathic hypertrophic pachymeningeal lesions: correlation between clinical patterns and neuroimaging characteristics. Eur Neurol. 1998; 39:49-56.

14. Martin N, Masson C, Henin D, Mompoint D, Marsault C, Nahum H. Hypertrophic cranial pachymeningitis: assessment with $\mathrm{CT}$ and $\mathrm{MR}$ imaging. AJNR Am J Neuroradiol. 1989; 10:477484. 